\title{
Eficiencia de la educación superior en Colombia: un análisis mediante fronteras*
}

\author{
Efficiency of higher \\ education in Colombia: \\ An analysys through borders
}

Iván Darío Rodríguez Murillo**

Recibido 20 de Abril de 2014

Revisado: 9 de Junio de 2014

Aprobado: 11 de septiembre de 2014

\section{Resumen}

Esta investigación evalúa el desempeño de las Instituciones de Educación Superior (IES) de Colombia, mediante la estimación de una Frontera de Posibilidades de Producción (FPP), que permite evaluar la eficiencia relativa y sus determinantes; también, se miden los factores externos que pueden influir en la calidad de la educación en Colombia. Para este fin, la metodología empleada es el Data Envelopment Analysis (DEA) y el análisis

* Agradezco a los profesores Álvaro Díaz Niño y Henry Laverde por su guía durante el proceso de elaboración de la tesis y sus invaluables comentarios y sugerencias. Las opiniones y posibles errores son responsabilidad exclusiva del autor y no comprometen ni al director, ni a la universidad. Documento publicado en la Serie Documentos de Trabajo CIELJL. Documentos de Trabajo de la Facultad de Economía de la Universidad Santo Tomás. Cómo citar este artículo: Rodríguez, I. (2014). Eficiencia de la educación superior en Colombia: un análisis mediante fronteras. Revista CIFE, 16(24), 163-194.

* Estudiante de Maestría en Ciencias Económicas, Universidad Santo Tomás. Correo: idrodriguez47@gmail.com 
ISSN: 0124-3551 / Año 16, No 24 / enero-junio / pp. 161-194

de fronteras estocásticas (FE). Los resultados sugieren que variables como el nivel socioeconómico de los estudiantes determina la calidad educativa.

Adicionalmente, se encuentra que la eficiencia de las IES vía DEA es en promedio 0,72, mientras que por medio de FE es de 0,87

Palabras clave: Educación superior, Frontera de Posibilidades de Producción, Data Envelopment Analysis, fronteras estocásticas.

Glasificación JEL: I23, L23, C14, C10

\section{Abstract}

This research evaluates the performance of Colombia's Higher Education Institutions (HEI), by estimating a Production Possibility Frontier (PPF) which allows the evaluation of the relative efficiency and its determinants, external factors that can influence the quality of the education in Colombia are also measured. For this purpose, the methodology employed is the Data Envelopment Analysis (DEA) and the Stochastic Frontier Analysis (SFA). Results suggest that variables such as the socio-economic status of students determines the quality of education.

Additionally, it is found that the efficiency of HEI through DEA is, in average, 0.72 , while done through SFA is 0.87 .

Keywords: Higher education, Production Possibility Frontier, Data Envelopment Analysis, Stochastic Frontier analysis.

Glassification JEL: I23, L23, C14, G10 


\section{Introducción}

Una amplia rama de la literatura que aborda la economía de la educación, particularmente relacionada con la calidad educativa, centra su análisis en el rendimiento de los estudiantes y no tiene en cuenta el papel que desempeñan las Instituciones de Educación Superior (IES), en cuanto a la provisión y el aprovechamiento de los recursos que conducen a la eficiencia de la producción académica.

En Colombia, las investigaciones empíricas realizadas para analizar los determinantes del desempeño educativo han presentado limitaciones, dado que no se ha estimado de manera robusta y comprensiva una Frontera de Posibilidades de Producción (FPP), lo cual no ha permitido realizar una comparación del rendimiento entre estudiantes y/o IES, es decir, las estimaciones econométricas tradicionales no permiten identificar una FPP.

Ante este vacío, el propósito de este documento es estimar una FPP educativa que permita evaluar el desempeño de las IES en términos de eficiencia y analizar sus determinantes. Además, se busca medir los factores externos que pueden influir en la calidad de la educación en Colombia.

Para estimar la FPP, se seguirán dos tipos de metodologías. En primer lugar, un método no paramétrico conocido en la literatura como DEA (Data Envelopment Analysis), el cual consigue estimar la FPP mediante técnicas de programación matemática, con la ventaja de no suponer a priori una forma funcional para la función de producción. En segundo lugar, se utilizará una metodología paramétrica que permite capturar los errores estocásticos en la estimación, de los cuales una parte será atribuida a la ineficiencia de las IES y la otra, a factores externos. Dada la concepción de ambas metodologías, se podrá dilucidar la consistencia y robustez de los resultados encontrados, por lo que estas se consideran complementarias.

El trabajo se divide en seis partes. En la segunda parte, se hace una revisión de la literatura existente sobre eficiencia de las IES y las fronteras de eficiencia. En la tercera, se describe la metodología a seguir para el cálculo de las FPP. En la cuarta, se presentan los resultados empíricos de la estimación por medio del DEA y de FE (fronteras estocásticas). La quinta parte corresponde a las conclusiones. Finalmente, se brindan varias recomendaciones para el mejoramiento de la calidad de la educación superior en Colombia, a partir de los resultados obtenidos.

Este documento no pretende medir los incrementos del conocimiento o las habilidades de los estudiantes al momento de elegir una IES, ni cuál de ellas es la mejor a la hora de incrementar las capacidades individuales. 


\section{Antecedentes}

En la literatura referente al rendimiento académico, existen dos enfoques importantes: uno orientado al estudio de los determinantes del rendimiento académico de los estudiantes y el otro encaminado a analizar el desempeño de las IES como productoras de servicios educativos.

Por una parte, en lo referente al estudio de los determinantes del rendimiento académico, los trabajos más conocidos son los de Hanushek (1986, 1989, 1991, 1996), Blundell (1997), Bets y Morrel (1999), y Neylor y Smith (2004), los cuales intentan analizar el efecto de determinadas características sobre una variable de desempeño educativo a través de modelos econométricos. Sin embargo, este tipo de estimaciones dejan de lado la posibilidad de identificar una FPP.

De otro lado, los estudios que elaboran el análisis del desempeño de las universidades estiman fronteras de producción, empleando métodos de eficiencia como el Análisis Envolvente de Datos (DEA, por sus siglas en inglés), o las fronteras estocásticas (FE), entre ellos Charnes et al. (1981), Bessent et al. (1980), Mc Carty y Yaisawanrg (1993), y Chalos (1997).

En el ámbito latinoamericano, el estudio de Miranda et al. (2003) evalúa la eficiencia del Programa para el Mejoramiento de la Calidad y el Cubrimiento de la Educación (MECE), en el área austral de Chile. Los resultados muestran que las instituciones ineficientes son aquellas con mayor cantidad de estudiantes y menor capacitación del docente. González et al. (2010) estiman una función de producción a partir de los datos de los anuarios estadísticos del Consejo de Rectores y del Consejo Superior de Educación de Chile, en los que encuentra evidencia que demuestra cómo el mayor financiamiento público en instituciones privadas implica tener mejores resultados de eficiencia; además, que las universidades públicas tienen resultados más eficientes que las privadas.

Para el caso de Argentina, Coria (2008) estudia la eficiencia técnica de las universidades de gestión estatal, en donde encuentra ineficiencias cercanas al $30 \%$ en total de unidades de decisión analizadas. Carrella (2009) evalúa, a través de FE y del DEA, la eficiencia de los estudiantes de las tres principales facultades de economía de Argentina, con lo que demuestra que son más eficientes aquellos alumnos que trabajan en el mismo campo en el que se formaron.

En México, Becerril-Torres et al. (2012) estiman una frontera de producción a través del método DEA, la cual demuestra que la eficiencia promedio de la educación en México está cercana al $70 \%$.

Para Colombia, el Departamento Nacional de Planeación (DNP) estimó una frontera de producción departamental a través del DEA. En ella, establece cómo la inversión en 
educación, los contratos privados y el número de docentes debe fomentar alumnos con mejores calificaciones. El estudio fue realizado para la educación media, en el que se demostraron resultados adversos en materia de eficiencia para los departamentos. Entre las debilidades de este estudio, se encuentra que el método utilizado no tiene en cuenta los errores estocásticos que se presentan en el proceso de aprendizaje.

Quesada et al. (2010) evalúan la eficiencia en la cobertura educativa en el departamento de Bolívar, y encuentran que tan solo el $30 \%$ de los municipios son eficientes en la prestación del servicio. García et al. (2013) hacen un análisis de eficiencia de las universidades públicas del país, teniendo en cuenta algunos índices construidos como la formación, la investigación y la capacidad. El estudio concluye que las universidades públicas más eficientes en investigación son la Universidad Nacional sede Antioquia y la Universidad del Valle, y que no existe una continuidad de eficiencia en el tiempo. Las debilidades de estos documentos radican en utilizar un número reducido de IES, debido a que las universidades privadas representan aproximadamente el $70 \%$ de las IES en Colombia.

En general, aunque existe literatura empírica de los determinantes del rendimiento académico y el desempeño de las universidades, para Colombia hay pocos estudios que estimen fronteras de producción para las IES. Además, este tipo de estudios han sido analizados a través de metodologías como el DEA, pero poco explorados por otro tipo de metodologías, como la de FE.

\section{Metodología}

Existen múltiples metodologías para analizar la eficiencia del sector educativo y sus determinantes. La literatura tradicional evalúa el desempeño académico tanto individual,

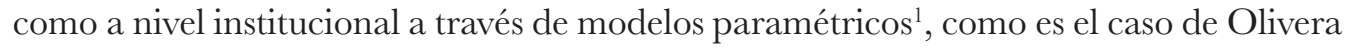
(1964), Hanushek (1979, 1986 y 1993), Coleman et al. (1966), Betts y Morell (1999), Naylor y Smith (2004), Porto et al. (2004) y Porto (2007), entre otros.

Pero determinar una forma funcional de la función de producción educativa tiene una implicación restrictiva grande, debido a que no se conocen los rendimientos propios del proceso de producción. De esta forma, las estimaciones por medio de mínimos cuadrados ordinarios evalúan la media de la función de producción y no la FPP².

Por esta razón, para estimar una FPP robusta, se propone utilizar dos metodologías que para el caso se consideran complementarias. La primera, conocida como Análisis

1 Comúnmente, el análisis se hace con base en regresiones de modelos econométricos, mediante Mínimos Cuadrados Ordinarios (MCO).

2 Es decir, al tener una función de producción dada, se intenta minimizar el error cuadrado de la estimación por medio de una regresión lineal y no se trabaja directamente sobre la frontera de posibilidades de producción. 
Envolvente de Datos (DEA), busca establecer la eficiencia relativa existente entre diferentes IES y así trazar una FPP guiada con los propios datos. La segunda, denominada fronteras estocásticas (FE), examina la eficiencia existente entre IES y normaliza los resultados, teniendo en cuenta las externalidades que intervienen en el proceso de producción educativa, por ejemplo, la suerte o el clima. Dichos métodos se encuentran dentro de los principales recursos empíricos de la literatura correspondiente a las fronteras de eficiencia, y sus principales características se esbozan a continuación.

\subsection{Análisis Envolvente de Datos (DEA)}

Uno de los primeros acercamientos al término de eficiencia técnica se encuentra en el documento de Farell (1953)3 , sin embargo, fueron Charnes, Cooper y Rhodes (1978) quienes completaron dicho trabajo, llevando a cabo un análisis más detallado de los supuestos de la medida de eficiencia de Farell y desarrollando una medición de la eficiencia técnica llamada DEA.

El DEA es un modelo determinístico, no estadístico y no paramétrico, que efectúa el análisis y la comparación de las diferentes IES mediante la construcción de una función de producción convex ${ }^{4}$, utiliza datos de insumos y productos, y trabaja bajo la lógica de la programación lineal, para así obtener un score de eficiencia relativa. Contrario al análisis tradicional de ajuste de tendencias, el DEA permite construir la FPP a partir de IES $^{5}$ eficientes.

Las estimaciones econométricas comunes tienen como fin optimizar los datos. El DEA optimiza cada observación y calcula una FPP óptima, la cual está determinada por las unidades de decisión eficientes, que a su vez cumplen con la condición de optimalidad de Pareto. Es necesario resaltar que la FPP cumple el principio de que un aumento en el producto no es posible sin disminuir otros insumos o productos ${ }^{6}$.

Entre las ventajas del DEA, se encuentra que no requiere ningún supuesto sobre la forma funcional de la FPP de las IES. Además, el DEA ofrece un mejor análisis de los datos, debido a que permite ver la ineficiencia de las otras IES con respecto a las mejores. Es por esto que la FPP es denominada "frontera de posibilidades de producción de mejor práctica", ya que calcula la eficiencia de cada agente maximizador en relación con las mejores IES.

3 Farell construyó una medida de eficiencia entendida como la relación de insumo-producto de las firmas. Para mayor detalle, ver Farell (1957).

4 La convexidad del DEA permite que los datos se envuelvan a través de una frontera trazada por las unidades eficientes, formando una FPP. Además, garantiza que no se violen los supuestos de la función de producción e incluye en las variables convexas del conjunto.

5 Las IES serán las Decision Making Units del presente estudio.

6 Para el caso, esta condición se cumple siempre y cuando cualquier cambio en los insumos no cambie los productos.

7 También se puede encontrar en la literatura como: Frontera de posibilidades de producción de eficiencia relativa u observada. 
Contrario a las estimaciones econométricas tradicionales, no es necesario correr un test de significancia estadística para los resultados, gracias a que son los mismos datos los que se encargan de evaluarse entre sí. De esta forma, las IES se considerarán agentes maximizadores, cuyo objetivo es alcanzar el máximo producto con los insumos provistos o en su forma dual, de modo que el costo unitario sea el menor posible.

El principal objetivo de utilizar el DEA en este documento es analizar la eficiencia relativa de las IES colombianas. Siguiendo a Seiford y Thall (1990), la eficiencia técnica relativa de las IES consiste en resolver un programa lineal para cada una de ellas, en el cual se buscan combinaciones lineales de otras IES que generen más productos con los insumos dados. En el caso de que no se pueda encontrar esa combinación, se entenderá que la IES es eficiente, y por supuesto, para un caso tal, el DEA provee una medida de eficiencia técnica que indica el aumento proporcional de los insumos si las IES fueran eficientes.

Cabe recordar que la medida de eficiencia relativa estará determinada por un valor entre 0 y 1 , en el que 0 es totalmente ineficiente y 1 es eficiente, por lo cual todas aquellas IES ineficientes obtendrán un score de eficiencia relativa menor que 1.

Siguiendo a Charnes et al. (1981): "La eficiencia se puede caracterizar dependiendo de dos direcciones posibles” (gráfico 1). La primera está orientada a los insumos y cada IES busca minimizar su utilización; es decir que serán eficientes solo aquellas IES que consigan la mayor cantidad de producto con el menor insumo. La segunda, por su parte, hace referencia a la maximización de los productos, y es la que se utiliza más a menudo en la literatura de fronteras: aquellas IES que generen mayor producto con la combinación de insumos más eficientes trazarán la frontera.

Gráfico 1. Fronteras de Eficiencia mediante DEA.
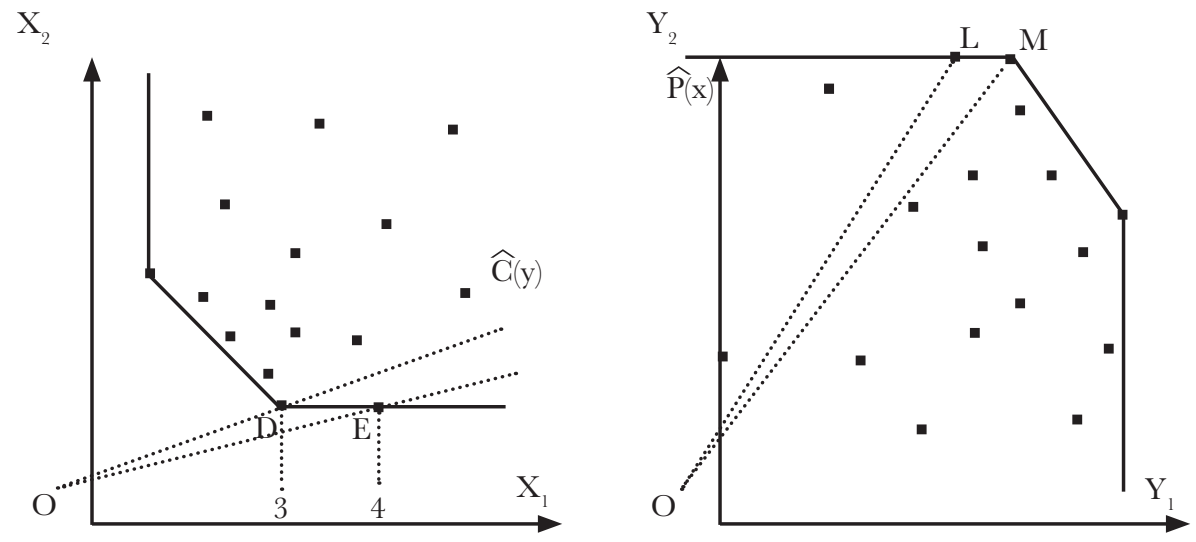

Fuente: Charnes, Cooper, Rhodes (1981). 
En este trabajo, el modelo DEA se construye según la lógica de un modelo orientado a la maximización del producto con rendimientos variables a escala, en el que se consideran $\mathrm{n}$ unidades de decisión, las cuales utilizan $\mathrm{m}$ insumos, con el fin de generar s productos. La programación lineal del DEA sigue el planteamiento de Carrella (2009) y se bosqueja como:

$$
\max _{\varphi, \lambda} \varphi
$$

S.A

$$
\begin{gathered}
\text { (1) } \sum_{j=1}^{n} \lambda_{j} Y_{r j} \geq \varphi_{k} Y_{r k} ; r=1, \ldots, s . \\
\text { (2) } \sum_{j=1}^{n} \lambda_{j} X_{i j} \leq X_{r k} ; i=1, \ldots, m . \\
\text { (3) } \sum_{j=n}^{n} \lambda_{j}=1 \\
\text { (4) } \lambda_{i} \geq 0
\end{gathered}
$$

Donde, yrk representa la cantidad de producto r que utiliza la $k$-ésima IES. Así mismo, xrk representa la cantidad de insumo i que utiliza la misma IES. La variable j representa a las otras IES, es decir, se comparan los insumos y productos de cada unidad de decisión con las demás. $\lambda$ es un vector de constantes $n x 1$, el cual será la ponderación asignada resultante del ejercicio de programación lineal. La condición para que el modelo trabaje bajo el supuesto de retornos variables a escala estará dada por la ecuación (3), y por último, el valor de $\varphi \mathrm{k}$ representa el valor el cual debe aumentar $\mathrm{y}_{\mathrm{rk}}$ para llegar a la relación convexa dominante y así poder ser frontera.

Con el fin de construir una FPP educativa, el producto será definido por el rendimiento académico, en este caso, las pruebas Saber Pro. Otros productos que se han utilizado en la literatura son el número de materias aprobadas, las pruebas internacionales y la producción investigativa. Como insumo suelen utilizarse características de los alumnos que explican ese rendimiento; por ejemplo, Betts y Morell (1999), y Porto y Di Gresia (200 1) encuentran que los elementos con mayor significancia a la hora de medir el desempeño académico son las características propias de los estudiantes.

El DEA compara los resultados obtenidos por las IES que tienen alumnos con características similares; es decir, la FPP será dada por las IES que tienen mayor puntaje en la prueba, dependiendo de las características de sus estudiantes, y el resto de IES tendrán un puntaje de eficiencia dado según sus dotaciones de insumos y productos. 
A modo de ejemplo ${ }^{8}$, suponiendo que solo existen dos IES: Aquí, A tiene un nivel de producto $\mathrm{Y}$ y un nivel de insumo $\mathrm{X}$ 1, mientras que $\mathrm{B}$ tiene un nivel de insumo $\mathrm{Y}^{\prime}>\mathrm{Y}$ y un producto $\mathrm{X}_{2}=\mathrm{X}_{1}$, entonces se asumiría que $\mathrm{B}$ es eficiente, dado que presenta mejores resultados con las mismas dotaciones iniciales, donde $\mathrm{B}$ obtiene un índice de eficiencia de la forma $\frac{\mathrm{Y}}{\mathrm{Y}^{\prime}}$

De esta manera, pueden existir IES eficientes que en realidad no lo son, debido a que al tratarse de eficiencia relativa, es necesario que existan otras unidades de comparación. Por ser un método de programación lineal que parte de los datos para trazar la FPP, el DEA no mide la capacidad potencial de las IES, sino la producción relativa a unidades homogéneas. Además, el DEA no mide los factores externos que pueden incidir en la eficiencia del proceso productivo, como la suerte o el clima, motivo por el cual esta metodología se complementa con un análisis de fronteras estocásticas (FE), que se describe a continuación.

\subsection{Análisis de frontera estocástica (FE)}

Aunque la FE como metodología para el estudio de la eficiencia en el sector educativo no ha sido tan utilizada como el DEA, sí ha sido trascendental en el análisis de los determinantes de la FPP. Como documento pionero de esta metodología, se encuentra Aigner, Lovell y Schmidt (1977). Además, se pueden destacar los estudios de Battese y Corra (1977), Segupta y Sfeir (1986), Segupta (1987), Barrow (1991), Deller y Rudnicko (1993), Cubbin y Zamani (1996), y Bates (1997), entre otros.

El análisis FE es un método estadístico, estocástico y paramétrico, que implica una función de producción para datos de corte transversal ${ }^{9}$ y cuenta con un error compuesto por: un componente aleatorio (V) y la ineficiencia técnica (U) (gráfico 2).

Por una parte, el componente aleatorio $(\mathrm{V})$ hace referencia a todos aquellos errores que se generan por especificación o factores externos al proceso productivo (azar o ruido); por ejemplo: la suerte de los estudiantes al presentar el examen Saber Pro, el estado de ánimo, etc. Por otra parte, el error causado por $(\mathbf{U})$ genera una desviación de la frontera de producción a causa de ineficiencia técnica y de asignación de recursos por parte de la IES.

8 Para el presente ejemplo, se presumen dos individuos con un solo producto y un solo insumo. Para el caso de varios insumos o productos, es necesario ponderar el valor de los insumos por su peso en el desarrollo del producto o viceversa.

9 La implicación de una función de corte transversal es debido a la naturaleza de esta investigación, sin embargo, existen extensiones que incluyen: distribuciones normales truncadas, two parameter gama, datos de panel y time varying. Para más información, ver Battese y Coelli (1992). 
Gráfico 2. Fronteras de producción estocástica.

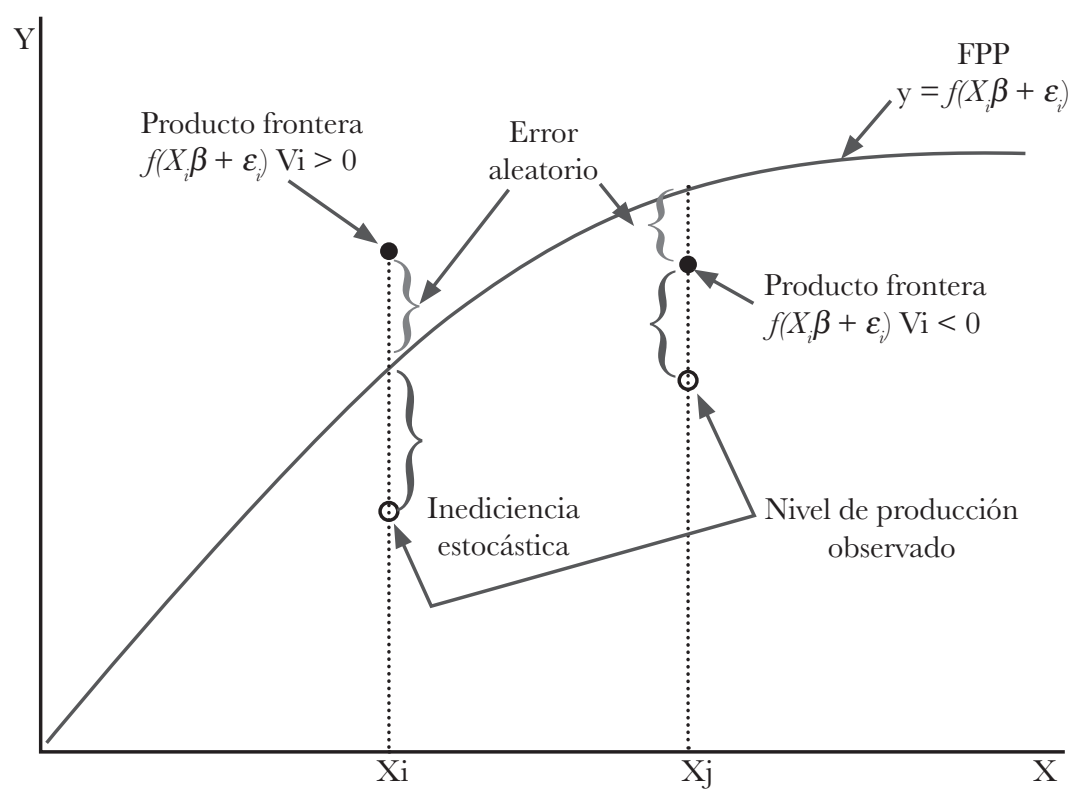

Fuente: elaboración propia a partir de: Coelli et al. (2005).

Se seguirá el supuesto de que los dos componentes de error tienen una estructura ineficiente que sigue una distribución de media normal, errores aleatorios ${ }^{10}$ y normalmente distribuidos.

Siguiendo a Aigner, Lovell y Schmidt (1977), el modelo se expresará como:

$$
\text { (5) } \log Y_{i}=\beta\left(X_{i}\right)+\left(V_{i}-U_{i}\right)
$$

Dónde:

$Y_{i}$ representa el producto de la IES $i$; Xi en un vector $K \mathrm{x} l$ cantidades de insumo; $b$ es el vector de los parámetros a estimar; $V i$ son los errores aleatorios no controlables (factores exógenos) y $U i$ son las variables aleatorias no negativas que miden la ineficiencia técnica en la producción.

De esta forma, se puede establecer gráficamente que la estimación de este método está dada por la distancia del error aleatorio más la ineficiencia estocástica.

10 Abarcan los eventos fuera del control de las IES. 
La FE permite diferenciar entre término de error aleatorio y factores exógenos,lo cual permite distar las desviaciones de la FPP dependiendo de las ineficiencias. Adicionalmente, se supone que la distribución es normal tanto para los errores exógenos como para la ineficiencia.

Una de las principales desventajas del análisis de FE es que supone una forma funcional para la función de producción. La elección de la función de producción particular puede tener restricciones severas con respecto a los tipos de análisis que se pueden realizar. Dicha forma funcional es difícil de medir, debido a que no se conocen los incrementos del conocimiento o las habilidades que tienen los estudiantes al momento de elegir una IES o cuál de ellas es la mejor a la hora de incrementar las capacidades individuales. Otra desventaja del análisis de FE es que al incorporar múltiples productos, la construcción de la frontera se torna complicada.

En los estudios de la función de producción por medio de FE, es usual encontrar como insumo las características de la enseñanza - capacidad docente, relación docente-alumno, tipo de educación y ambiente de aprendizaje (áreas, bibliotecas, tecnología)-. Los productos más utilizados hacen referencia a los test de suficiencia y a la capacidad de producción de artículos académicos. En esta línea, se puede encontrar, por ejemplo, a Deller y Rudnicki (1993).

\subsection{Datos}

Los datos utilizados en este documento provienen del Ministerio de Educación Nacional $(\mathrm{MEN})^{11}$ y del Instituto Colombiano para el Fomento de la Educación Superior (Icfes).

En particular, se utilizan datos de docentes clasificados por grado de educación y costo promedio de la matrícula, así como por factores socioeconómicos del promedio de estudiantes de las IES. Por último, se analizan los resultados promedio de las pruebas de núcleo común del examen Saber Pro por IES, los cuales son facilitados por el Icfes.

Se estudia el rendimiento académico y la calidad de la educación para una cohorte de alumnos que ingresaron en el 2006 y terminaron sus estudios en 2011. Se elige esta cohorte por ser la información más reciente en cuanto a datos disponibles y porque asegura que no se hayan realizado cambios significativos en el sistema educativo. La tabla 1 hace un recuento de las características primordiales de la muestra seleccionada.

11 El MEN tiene bases de datos especializadas que se dividen, según el criterio del investigador, en: Sistema Nacional de Evaluación Docente de la Educación Superior (SNEDES), Sistema Nacional de Información de la Educación Superior (SNIES), Sistema para la Prevención de la Deserción de la Educación Superior (SPADIES), Sistema de Aseguramiento de la Calidad de la Educación Superior (SACES) y el Observatorio Laboral para la Educación. 
Tabla 1. Características de la muestra.

\begin{tabular}{|l|c|c|}
\hline & Total & Participación \\
\hline Alumnos & 96264 & \\
\hline Edad promedio & 27 & \\
\hline$\%$ Hombre & 41394 & $43 \%$ \\
\hline$\%$ Mujeres & 54870 & $57 \%$ \\
\hline Total IES & 281 & \\
\hline$\%$ U publicas & 95 & $34 \%$ \\
\hline$\%$ U privadas & 186 & $66 \%$ \\
\hline
\end{tabular}

Fuente: elaboración propia a partir de datos ICFES y MEN.

En total, se analizan 281 IES $^{12}$, con un total de 96.264 personas que presentaron el examen de estado de calidad de la educación superior, Saber-pro.

La edad promedio de los estudiantes que presentaron el examen es de 27 años, esto se explica porque se evalúan estudiantes de jornada diurna y nocturna, lo cual aumenta el promedio de edad. Del total de estudiantes, el 57 \% son mujeres, lo cual corrobora los datos utilizados por Forero y Ramírez (2008, pp. 16) y deja abierta la discusión hacia la eficiencia por género.

En Colombia, trabajos anteriores como el García et al. (2013) evalúan la eficiencia de la educación pública; sin embargo, no tienen en cuenta las IES privadas. Este es otro aspecto importante en el presente estudio, pues toma en cuenta las IES privadas, que corresponden al $66 \%$ de las 281; tan solo el $34 \%$ restante es de carácter público.

En la literatura de la economía de la educación, se han utilizado múltiples variables para explicar la calidad y el desempeño académico. Entre las variables endógenas más usadas, se encuentran las pruebas de Estado y las pruebas internacionales ${ }^{13}$. Otros trabajos como el de Smith et al. (1987) han utilizado otro tipo de producto, con resultados importantes acerca de la eficiencia en el nivel de abandono de las actividades escolares.

De otro lado, las variables exógenas utilizadas van en doble vía: la primera sitúa aquellos estudios que buscan evaluar la eficiencia económica y la segunda, aquellos que tienen en cuenta el entorno socioeconómico de los estudiantes.

12 Se entienden como IES las instituciones universitarias, tecnológicas profesionales, tecnológicas terminales y normalistas, las cuales tienen modalidad presencial, semipresencial y a distancia.

13 Entre las pruebas internacionales más usadas, se encuentran: CIVET, LLECE, PIRLS, PISA y TIMSS.. 
Estudios como el de González (2010) y el de Murias et al. (2008) buscan la eficiencia económica minimizando el gasto público en educación. Los resultados empíricos demuestran que existe una relación causal positiva entre el gasto público en educación y la eficiencia de las IES.

Cuando se evalúa la eficiencia económica, se tiende a dejar de lado la calidad educativa, y por lo tanto, se sacrifica la asignación de otros recursos como planta docente o la situación socioeconómica de los estudiantes.

De tal forma, se puede decir que la mayoría de estudios desarrollados con base en metodologías como el DEA y el MCO no toman en cuenta factores como la suerte y el clima. Debido a esto, se crea la necesidad de ver más allá del gasto público en educación y entrar a diferenciar variables aleatorias y socioeconómicas que determinan la eficiencia de las IES.

Lo anterior se debe a que la calidad de vida, el nivel educativo de los padres y el estrato social, entre otros, son aspectos relevantes en el rendimiento educativo. Por ejemplo, Segupta et al. (1988) y Deller et al. (1993) encuentran que el nivel educativo de los padres determina mejores resultados en las pruebas de Estado. Por tal razón, el presente estudio pretende analizar la calidad educativa de las 281 principales IES, sujetas a la calidad docente y a la situación socioeconómica de sus estudiantes, siempre y cuando al menos uno de ellos haya presentado el examen Saber Pro en el 2011.

Con el fin de medir la calidad de la educación en Colombia, se han generado las siguientes variables, que buscan medir la eficiencia técnica de las IES en Colombia y estimar una FPP viable, sin dejar de lado la parte social de los estudiantes.

El producto será el promedio ponderado de los resultados de los estudiantes de las diferentes IES en las pruebas Saber Pro ${ }^{14}$. En cuanto a los insumos a tener en cuenta, se tienen:

1. El número de estudiantes que presentaron la prueba.

2. La edad promedio de los estudiantes que presentaron la prueba.

3. Estado civil; se genera una variable Dummy que describe el estado civil ( 0 casado, 1 soltero).

4. Valor promedio de la matrícula (únicamente el valor que el estudiante reportó como último pago).

14 Se toma el promedio ponderado de las pruebas genéricas o de núcleo común, dado que estas son pruebas homogéneas para todas las IES. En estas pruebas, se miden la comunicación escrita, el razonamiento cuantitativo, la lectura crítica, las competencias ciudadanas y el inglés. No se toman los puntajes de las pruebas específicas debido a la naturaleza del documento. 
ISSN: 0124-3551 / Año 16, No 24 / enero-junio / pp. 161-194

5. Personas promedio que viven con los estudiantes.

6. Promedio de personas a cargo.

7. Años de educación de los padres (madre y padre).

8. Estrato social promedio de los estudiantes.

9. Ingreso familiar promedio

10. Número de profesores.

En la presente investigación, se mide la relación existente entre los insumos y los productos, y se busca establecer una FPP que permita identificar la producción óptima (de mejor práctica). La elección de las variables obedece entonces a la evidencia empírica existente como determinante del rendimiento y la calidad educativa.

En general, para entender más a fondo la relación de las variables que se van a utilizar, la tabla 2 muestra la estadística descriptiva de las variables endógenas y exógenas.

En la mayoría de las IES, predominan los educadores hombres. En promedio, por universidad hay 230 docentes hombres y 123 mujeres; es decir, las mujeres representan en promedio el $35 \%$ de la planta docente del país. En cuanto al nivel de preparación de los docentes, en promedio solo el $8 \%$ de los catedráticos a nivel nacional tienen un título de maestría y un $2 \%$ alcanza un doctorado; además, el nivel máximo de doctorados en IES es el $4 \%$ del total, mientras que en maestría alcanza el $61 \%$.

El valor promedio de la matrícula en las diferentes IES es de $\$ 2.500 .000$; sin embargo, este valor es el promedio del valor que el estudiante reportó como último pago y no el valor real del programa. Hasta este punto, la IES puede sesgar las variables para mejorar la calidad educativa, todo lo demás es constante, es decir, puede contratar personal más calificado o aumentar el valor de la matrícula.

De otro lado, al analizar las variables socioeconómicas, se encuentra que la edad promedio de la presentación del examen es de 27 años; además, el estrato del promedio de los estudiantes es 3 y se supone que los estudiantes son de estrato medio ${ }^{15}$. Los ingresos del hogar de los estudiantes son, en promedio, \$3.000.000, lo cual también hace más sólido este argumento. Así mismo, cerca del 90 \% de los estudiantes son solteros. Por último, siguiendo a Ibarreta et al. (2009), se incluye el nivel de educación de los padres, el cual es mayor en la madre que en el padre y oscila entre los 11 y 12 años de educación.

15 Estudios de diferentes universidades en cuanto al perfil de los estudiantes en Colombia validan esta información. La Universidad Autónoma de Occidente encuentra que sus estudiantes, en promedio, son estrato 2,8. Pérez et al. (2002) demuestran cómo el estrato de los estudiantes incide en el momento de decidir la universidad, por la capacidad de pago, y en los resultados de las pruebas. 
Tabla 2. Estadística Descriptiva.

\begin{tabular}{|c|c|c|c|c|}
\hline Variable & Mean & Std.Dev & Min & $\operatorname{Max}$ \\
\hline \multicolumn{5}{|l|}{ Docentes } \\
\hline Mujeres & 123 & 0.19 & 0 & 2494 \\
\hline Hombres & 231 & 0.33 & 0 & 4036 \\
\hline \multicolumn{5}{|l|}{ Docentes } \\
\hline$\%$ Maestria & 0 & 0.12 & 0 & 0.61 \\
\hline$\%$ Doctorado & 0 & 0.06 & 0 & 0.045 \\
\hline \multicolumn{5}{|l|}{ No estudiantes } \\
\hline Hombres & 193 & 299 & 0 & 2191 \\
\hline Mujeres & 145 & 221 & 0 & 1624 \\
\hline Vr matricuma prom & 2503636 & 1288821 & 0 & 5000000 \\
\hline Edad promedio & 27 & 6 & 19 & 81 \\
\hline No personas hogar & 4 & 0.6 & 0 & 6 \\
\hline No personas a cargo & 1 & 0.5 & 0 & 2 \\
\hline Estrato promedio & 3 & 0.7 & 1 & 6 \\
\hline Ingreso hogar & 2889482 & 897932 & 0 & 6018182 \\
\hline Dummy estado civil & 1 & 0 & 0 & 1 \\
\hline Años de educa madre & 12 & 2 & 0 & 24 \\
\hline Años de educa padre & 11 & 2 & 0 & 16 \\
\hline Puntaje saber pro & 52 & 9 & 19.15 & 67.87 \\
\hline Sostenibilidad total & 1.57 & 2.13 & 2.69 & 3.05 \\
\hline
\end{tabular}

Finalmente, en cuanto al producto se evidencia una mayor dispersión en lo referente a los resultados de las competencias genéricas de la prueba Saber Pro. Allí, la media en los componentes básicos es de 52 puntos, con un máximo de 67 y un mínimo de 19 .

Es así como se evidencia que las IES son homogéneas. La desviación estándar denota que los datos no están dispersos unos con otros, lo que da consistencia para la estimación de una FPP educativa robusta, que sirve como base para el análisis de la eficiencia técnica de las IES en Colombia. 


\section{Análisis Envolvente de Datos (DEA)}

Para la estimación del modelo, se dividió la muestra de acuerdo con el origen de su financiamiento (privado y público) y el carácter académico de las diferentes IES, según lo establecido en la Ley 30 de $1992^{16}$. Esto con el fin de garantizar la homogeneidad en la muestra seleccionada y garantizar estimadores más eficientes. A continuación, se describe el modelo DEA planteado.

El DEA es un método de programación lineal no paramétrico para asignar eficiencia y productividad a diferentes unidades de decisión (Lee et al., 2010) ${ }^{17}$. Para el modelo DEA, se relaciona como variable producto el resultado de las pruebas genéricas del examen Saber Pro por IES, mientras que como insumo están: el total de docentes, el número de estudiantes, el estrato ${ }^{18}$ y los años de educación promedio de los padres por IES. Se eligieron estas variables debido a que presentan la generalidad de las IES en cuanto a personal, origen, selección de estudiantes y entorno socioeconómico.

Los resultados de la estimación de la FPP educativa, en función de las variables mencionadas, se presentan en la tabla 3 .

En razón de la naturaleza del modelo, en cada grupo existe al menos una IES eficiente. Sin embargo, el número total de IES eficientes es similar en aquellas de carácter público que en las privadas. La eficiencia mínima es análoga para todas las IES, independientemente de su carácter académico, con excepción de las instituciones tecnológicas privadas y las instituciones universitarias públicas, las cuales presentan scores mínimos mucho más altos.

Es importante prestar atención a este resultado, pues puede malinterpretarse. No significa que la IES menos eficiente de estos grupos sea más eficiente que las de los demás grupos; es decir, al dividir la muestra, se traza una frontera de eficiencia para cada una de las IES, dependiendo de su carácter académico, lo que implica que el resultado de cada grupo es comparable únicamente con la IES eficiente dentro del mismo grupo.

A modo de ejemplo, el Grafico 3 ilustra la frontera estimada para las instituciones tecnológicas públicas. Aquí se evidencia como la eficiencia se denota solo en su conjunto de datos, por lo cual resulta imposible de comparar los resultados entre las IES de distinto

16 En el capítulo V, Artículo 16, se establece que se reconocen como IES: las instituciones técnicas, tecnológicas profesionales, instituciones universitarias y universidades.

17 El software utilizado para asignar eficiencia técnica de las IES en Colombia será STATA®. La diferencia entre

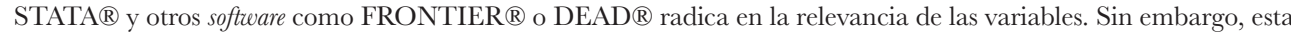
diferencia no presenta variaciones significativas en el resultado.

$18 \mathrm{Al}$ tener en cuenta el estrato, no se toman en cuenta el valor de la matrícula y los ingresos de la familia, dado que estas variables están altamente correlacionadas y generarían ruidos y problemas de estimación de la FPP. 
carácter educativo, sin embargo, si se permite tener un índice de eficiencia promedio de la educación superior.

Tabla 3. Estadística Descriptiva de las puntuaciones de eficiencia.

\begin{tabular}{|c|c|c|c|c|c|c|c|c|}
\hline Origen & Carácter & Obs & Media & Mediana & $\begin{array}{c}\text { Desv. } \\
\text { Est. }\end{array}$ & Min & Max & Dmueficientes \\
\hline \multirow{4}{*}{ Publicas } & $\begin{array}{l}\text { Institucion } \\
\text { universitaria }\end{array}$ & 21 & 0.82 & 0.88 & 0.18 & 0.52 & 1 & 5 \\
\hline & $\begin{array}{c}\text { Tecnica } \\
\text { profesional }\end{array}$ & 36 & 0.58 & 0.59 & 0.2 & 0.33 & 1 & 3 \\
\hline & $\begin{array}{l}\text { Institucion } \\
\text { tecnologica }\end{array}$ & 7 & 0.73 & 0.69 & 0.23 & 0.34 & 1 & 2 \\
\hline & Universidad & 31 & 0.81 & 0.74 & 0.17 & 0.39 & 1 & 13 \\
\hline \multicolumn{2}{|c|}{ Total publicas } & 95 & 0.74 & 0.73 & 0.20 & 0.40 & 1 & 23 \\
\hline \multirow{4}{*}{$\begin{array}{l}\text { Priva- } \\
\text { das }\end{array}$} & $\begin{array}{c}\text { Institucion } \\
\text { universitaria }\end{array}$ & 76 & 0.69 & 0.69 & 0.19 & 0.23 & 1 & 9 \\
\hline & $\begin{array}{c}\text { Tecnica } \\
\text { profesional }\end{array}$ & 30 & 0.70 & 0.75 & 0.17 & 0.27 & 1 & 3 \\
\hline & $\begin{array}{l}\text { Institucion } \\
\text { tecnologica }\end{array}$ & 35 & 0.69 & 0.6 & 0.17 & 0.51 & 1 & 4 \\
\hline & Universidad & 45 & 0.74 & 0.71 & 0.15 & 0.4 & 1 & 6 \\
\hline \multicolumn{2}{|c|}{ Total privadas } & 186 & 0.71 & 0.69 & 0.17 & 0.35 & 1 & 22 \\
\hline
\end{tabular}

Para este caso, las IES eficientes son: la Escuela Superior Tecnológica de Artes y la Unidades Tecnológicas de Santander (UTS), pero independientemente de que sean eficientes en su grupo, pueden ser ineficientes con respecto a los demás grupos.

De otro lado, en lo referente a la eficiencia promedio, los grupos que presentan resultados más altos son las IES públicas y privadas, aunque las públicas son las que en promedio son más eficientes, con un score de eficiencia promedio de 0,74 frente a 0,71 de las IES privadas.

Así, se puede decir que el grupo de IES públicas presenta resultados más cercanos a la eficiencia. Además, en promedio la eficiencia de las IES en Colombia es de 0,72, con un promedio mínimo de 0,38, lo cual prevé una ineficiencia relativa del $28 \%$. Por último, 


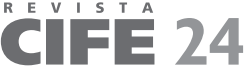

ISSN: 0124-3551 / Año 16, No 24 / enero-junio / pp. 161-194

es preocupante ver cómo del total de la muestra tan solo el $16 \%(45)$ corresponde a IES eficientes.

Los resultados obtenidos mediante el análisis DEA resultan convenientes para hacer un esbozo de la eficiencia relativa de la educación superior en Colombia, un análisis no condicionado sobre las medidas de eficiencia en cada grupo. Sin embargo, queda descubierto el tema de la robustez del modelo y el análisis de los determinantes de la educación, lo que deja abiertas preguntas como: ¿es el estrato socioeconómico un determinante en la eficiencia de las IES?, ¿cómo influye la educación de los padres en los resultados de las IES en las pruebas Saber Pro?, ¿qué impacto tiene el tamaño del hogar de los alumnos en la eficiencia de las IES?

Con el fin de responder estas preguntas, se hace la medición del efecto de estos factores sobre los indicadores de eficiencia de las IES. Cabe recordar que el score de eficiencia que se encontró mediante el análisis DEA se encuentra en un rango entre $[0,1]$, por lo cual el análisis debe tratarse mediante un modelo Tobit.

\section{Gráfico 3. IES Públicas tecnológicas.}

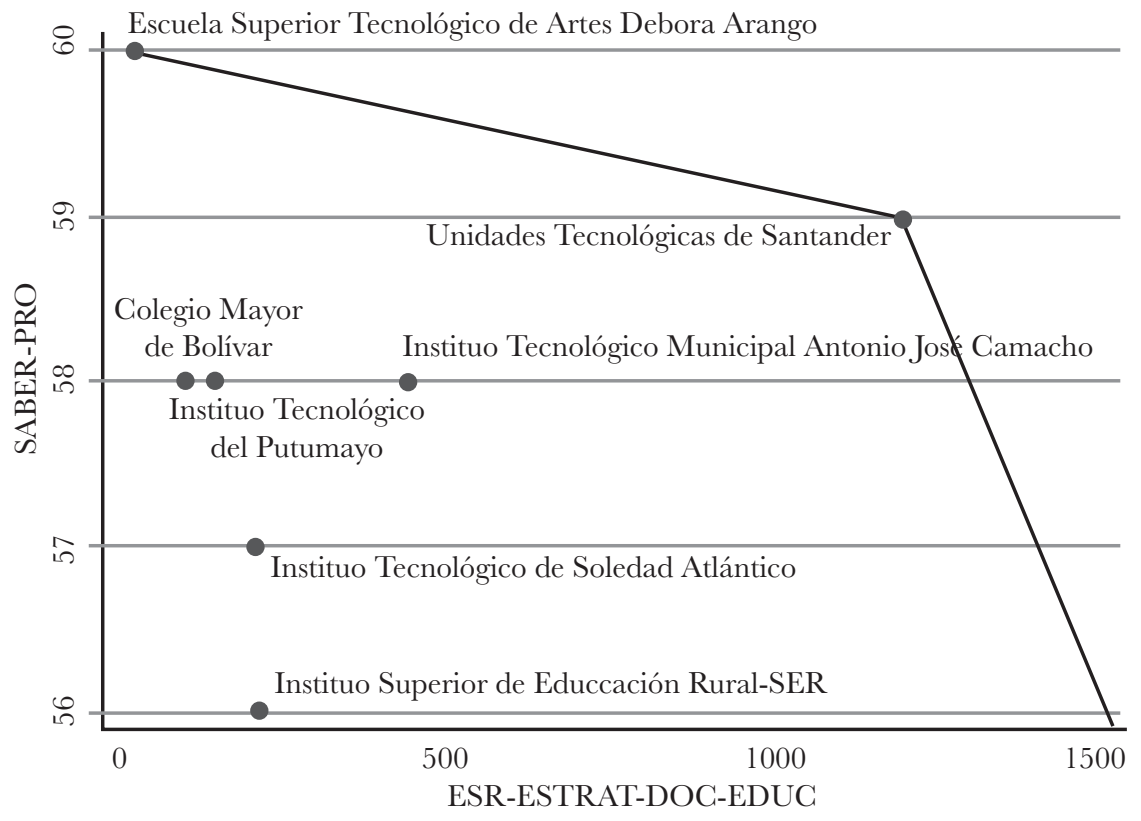

Fuente: cálculos propios en base a ICFES y MEN 


\section{Modelo Tobit}

La identificación de la eficiencia técnica por medio del DEA no permite medir los choques generados por agentes externos, ni validar la significancia estadística de las variables a medir. Así mismo, no es posible hacer ningún análisis de cara a los aspectos sociales y de género, que en muchas ocasiones provocan distorsiones en los resultados. Por esta razón, resulta importante realizar algunas consideraciones acerca de la variable dependiente. La variable dependiente se encuentra censurada a la derecha y a la izquierda. Debido a la construcción del modelo DEA, la eficiencia relativa esta denotada entre 0 y 1, con 1 como la unidad eficiente y 0 como la ineficiente. El hecho de que la variable dependiente se encuentre censurada provoca que al realizar la estimación por medio de MCO, los estimadores no cumplan la condición MELI ${ }^{19}$. Por esta razón, el mejor método a utilizar es el Tobit.

Siguiendo a Greene (2002), para la estimación de un modelo Tobit, la formulación general está expresada como:

$$
\begin{gathered}
\text { (6) } Y_{i}^{*}=X_{i}^{\prime}+\varepsilon_{i} \\
\text { (7) } Y_{i}=0 \rightarrow S I \rightarrow Y_{i}^{*} \leq 0 \\
\text { (8) } Y_{i}=1 \rightarrow S I \rightarrow Y_{i}^{*} \geq 1 \\
\text { (9) } Y_{i}=Y_{i}^{*} \rightarrow S I \rightarrow Y_{i}>0 " y^{\prime \prime} Y_{i}^{*}<1
\end{gathered}
$$

Donde $Y_{i}^{*}$ es la variable índice o variable latente (Greene, 2002, pp. 789). Además, $\Upsilon_{i}$ es la variable dependiente observada (scores de eficiencia), la cual tomará un valor entre $0 \mathrm{y}$ 1. Por su parte, $X_{i}$ contiene las variables explicativas (características de los docentes y de los estudiantes $)^{20}$ y por último el termino $\varepsilon_{\mathrm{i}}$ es el error.

Debido a que se quiere comparar la eficiencia entre las IES públicas y privadas, las estimaciones se realizan por separado. Los resultados de dichas estimaciones se muestran en la tabla 4.

Se debe tener en cuenta que al separar la muestra entre IES privadas y públicas, los coeficientes no son comparables entre sí. Además, puede que el coeficiente sea significativo para las IES privadas y no significativo en las públicas. A modo de ejemplo, el coeficiente del indicador de educación del padre es positivo y significativo para las IES privadas, pero puede ser no significativo en las públicas, lo cual se debe interpretar como: un

19 Hace referencia al mejor estimador lineal insesgado.

20 Las características de los estudiantes están enmarcadas en la parte socioeconómica y familiar. 
aumento en los años de educación de los padres genera un aumento en la eficiencia promedio de la IES privada. Así mismo, no se incluye como determinante el carácter académico de la institución, debido a que esta es una condición para armar la frontera y no dará información relevante de la eficiencia de la educación en Colombia.

En cuanto a la interpretación de los coeficientes, se puede decir que: las IES privadas que tienen vinculado un mayor número de docentes con maestría o doctorado son en promedio más eficientes. De hecho, si se toma como determinante solo los docentes con doctorado, la eficiencia es mayor; para las IES públicas, el coeficiente de docentes con doctorado es no significativo.

De igual modo, acorde con los resultados obtenidos por Carrela (2009), Betts y Morell (1999), y Naylor y Smith (2004) $)^{21}$, las IES que tienen más estudiantes mujeres son en promedio más eficientes que aquellas que tienen más hombres.

Con respecto a la educación de los padres, se puede establecer que mientras el nivel educativo del padre sea más alto, el nivel de eficiencia de la IES será menor. Esto es así porque a medida que aumentan los años de educación del padre, los resultados de las pruebas Saber Pro tienen mayor variabilidad. A su vez, los estudiantes de IES cuyos padres cuentan con niveles educativos bajos también tienen rendimientos promedio similares, y por lo tanto, no se encuentran indicadores de eficiencia extremadamente bajos. La disparidad de los resultados en aquellas IES que tienen estudiantes con padres, en promedio, más instruidos presenta un mayor rango de variabilidad para los índices de eficiencia de las IES.

Uno de los principales temas en el estudio de los determinantes de la eficiencia en las IES es el perfil socioeconómico de los estudiantes. Sin duda, se puede establecer que el tipo de hogar es uno de los determinantes a la hora de obtener mejores resultados.

Primero, entre más grande es el hogar del estudiante, menor es el resultado obtenido en las pruebas de Estado, lo cual afecta más a las IES públicas que a las privadas. Esto último puede justificarse como un comportamiento normal a la hora de presentar las pruebas, debido a que las responsabilidades adicionales al estudio varían. Por ejemplo, si el estudiante de la IES tiene una familia compuesta únicamente por sus padres, se esperaría que el tiempo promedio utilizado para estudiar fuera mayor que en el caso de aquel estudiante que tiene hermanos menores. Esto dado que, en la mayoría de los casos, quienes tienen dos o más hermanos también tienen menor espacio en su hogar para el estudio. Igualmente, la evidencia empírica ha demostrado que a medida que hay más integrantes en el hogar, los recursos financieros son menores en promedio por persona.

21 Estos trabajos han sido enfocados en la eficiencia por género y en todos se ha evidenciado que las mujeres obtienen en promedio mejores resultados que los hombres. 
Tabla 4. Resultados TOBIT para índices de eficiencia.

\begin{tabular}{|c|c|c|}
\hline & Privadas & Publicas \\
\hline Constante & 0.5426 & 1,121 \\
\hline & $(-0.0846)$ & $(0.5508)$ \\
\hline
\end{tabular}

\section{A. Docentes}

\begin{tabular}{|c|c|c|}
\hline \multirow[t]{2}{*}{ Con maestria } & $0.0064^{* *}$ & $0.0167 * * *$ \\
\hline & $(0.0075)$ & $(0.0165)$ \\
\hline \multirow{2}{*}{ Con doctorado } & $0.0126^{*}$ & -0.0045 \\
\hline & $(0.0094)$ & $(0.0219)$ \\
\hline \multicolumn{3}{|l|}{ B. Estudiantes } \\
\hline \multirow[t]{2}{*}{ Mujeres } & $0.0054 * * *$ & $0.0354 * *$ \\
\hline & $(0.0118)$ & $(0.0181)$ \\
\hline \multirow[t]{2}{*}{ Hombres } & $0.0014 * *$ & $0.0096 * * *$ \\
\hline & $(0.0115)$ & $(0.0203)$ \\
\hline \multicolumn{3}{|c|}{ C. Educacion de los padres } \\
\hline \multirow{2}{*}{ Padres } & -0.0237 & $0.0986^{* *}$ \\
\hline & $(0.0443)$ & $(0.0522)$ \\
\hline \multirow[t]{2}{*}{ Madre } & $0.0347 *$ & $0.0813^{* * *}$ \\
\hline & $(0.0508)$ & $(0.2076)$ \\
\hline \multicolumn{3}{|l|}{ D. Socio-economico } \\
\hline \multirow[t]{2}{*}{ Tamaño de hogar } & $-0,0239 * *$ & $-0,6704^{*}$ \\
\hline & $(0.0597)$ & $(0.1714)$ \\
\hline \multirow{2}{*}{ Valor de la matricula } & $0.0093^{* *}$ & $-0,0005^{* * *}$ \\
\hline & $(0.0047)$ & $(0.005)$ \\
\hline \multirow[t]{2}{*}{ Estado civil } & $0.0208 * * *$ & $0.0540 *$ \\
\hline & $(0.0250)$ & $(0.0322)$ \\
\hline \multirow[t]{2}{*}{ Personas a cargo } & $-0,0219 *$ & $-0,0032 * *$ \\
\hline & $(0.0117)$ & $(0.0213)$ \\
\hline Lr chi2(10) & 34.32 & 48.02 \\
\hline Prob>chi2 & 0.0002 & 0 \\
\hline Observaciones & 186 & 95 \\
\hline Censuradas izquierda & 1 & 1 \\
\hline Censuradas Derecha & 22 & 22 \\
\hline No censuradas & 163 & 71 \\
\hline
\end{tabular}

Fuente: cálculos propios en base a resultados DEA.

Nota: Los resultados tienen como variable dependiente la variable observada y no la latente, por lo cual, no se hace ponderación para mirar el efecto marginal del coeficiente en los resultados.

Error estándar entre paréntesis, * significativo al $1 \%$, **5 \%, *** $10 \%$ 
Segundo, aquellas IES cuyos estudiantes tienen en promedio más personas a cargo son más ineficientes. Por supuesto, podría pensarse que pasa igual que con el tamaño del hogar; sin embargo, este puede estar compuesto por hermanos y padres, mientras que al medir las personas a cargo se está haciendo referencia a los hijos, y además a los padres o hermanos con discapacidad, lo cual reduce el tiempo de estudio y se presenta como un problema de elección racional, con un costo de oportunidad dado. Por su parte, contrario a lo que se podría pensar, el estado civil tiene un coeficiente positivo que denota que si las instituciones tienen en promedio más estudiantes comprometidos sentimentalmente, son en promedio más eficientes.

Por último, en el grupo de las IES privadas, el valor de la matrícula influye positivamente en la generación de estudiantes con mayores fortalezas, debido a que las IES que se encuentran dentro de las fronteras son aquellas que tienen costos de matrículas más altos. Por ejemplo, en el grupo de estas universidades encontramos, entre otras, a la Universidad de los Andes, la Universidad Javeriana y la Universidad Eafit.

Teniendo en cuenta que las variables involucradas en el modelo DEA pueden ser limitadas, bien sea por problemas en su elección o por choques externos, se hace la estimación de una frontera estocástica que mide la ineficiencia a causa del aprovechamiento de los insumos y de otros factores.

\section{Análisis de Frontera Estocástica (FE)}

Debido a la limitación del DEA en la identificación de la ineficiencia ocasionada por factores externos y teniendo en cuenta que los índices de eficiencia técnica hasta este momento solo están denotados por los datos, es necesario validar que los resultados sean lo más robustos posible. Dicha robustez se evidencia en la significancia estadística que proveen las fronteras estocásticas.

La eficiencia asignativa garantiza que los choques externos sean tenidos en cuenta en la generación de una FPP y que, adicionalmente, se pueda validar el efecto real de los diferentes insumos en la generación de la calidad educativa. El modelo FE se basa en que las desviaciones de la frontera no están en total control de la unidad de estudio, contrario al DEA, debido a la existencia de factores externos que hacen que se alejen de su potencial. De hecho, el modelo trabaja bajo el supuesto de que cada IES enfrenta una frontera individual, la cual está determinada por su propia frontera de producción en función de los factores estocásticos que se incluyen en el modelo y aquellos que están fuera del control del agente.

Para el caso, cada IES se enfrenta a una FPP que determina su desempeño en la generación de calidad educativa. Sin embargo, existen elementos exógenos que hacen que la 
IES se aleje del rendimiento máximo esperado, sin importar que esté siendo eficiente en el uso de sus recursos; es decir, así la IES sea eficiente, podría ser mejor.

La estimación de la frontera estocástica para este estudio sigue los parámetros establecidos por Battese y Corra (1977), puesto que obedece a una tecnología de producción Cobb-Douglas, la cual se supone conforme con una distribución seminormal (exponencial) y es estimada por el método de máxima verosimilitud, que considera un patrón de variación común para todas las IES.

Para entender cómo se comporta la FPP en el análisis de FE, es necesario tener en cuenta lo siguiente:

Los coeficientes reportados en el modelo se pueden interpretar como en los modelos de MCO, es decir, el signo - positivo o negativo- del coeficiente indica el efecto que la variable exógena tendrá en la variable dependiente (calidad de la IES).

De otro lado, es necesario considerar que las FE tienen en cuenta un error compuesto por dos componentes, que están divididos en: un componente aleatorio $(\mathbf{V})$ y la ineficiencia técnica $(\mathrm{U})$.

El componente aleatorio $(\mathrm{V})$ hace referencia a los errores que se generan por especificación o a factores externos al proceso productivo. A su vez, el error causado por $(\mathbf{U})$ genera una desviación de la frontera de producción a causa de ineficiencia técnica y de asignación de recursos por parte de la IES. Se seguirá el supuesto de que los dos componentes de error tienen una estructura ineficiente que sigue una distribución de media normal, errores aleatorios (abarcan los eventos fuera del control de las IES) y normalmente distribuida.

Los resultados de la estimación de las FE para la calidad de la educación en Colombia con respecto a las pruebas Saber Pro se presentan en la tabla 5. La muestra se divide en públicas y privadas, al igual que en el modelo Tobit. Los resultados reportados no son directamente comparables con los de la tabla 4, debido a que las variables dependientes son diferentes ${ }^{22}$.

En cuanto al análisis de los coeficientes, tiene resultados muy parecidos con los determinantes de la eficiencia hallados mediante el modelo Tobit. Se encuentra que aquellas IES, independientemente del origen de la financiación, que tienen mayor número de docentes con maestría obtienen mejores resultados en las pruebas de calidad. Así mismo, para el caso de las IES privadas, el nivel de educación de los docentes

22 Para el modelo Tobit, la variable dependiente era la eficiencia de las IES encontrada en la estimación de la frontera vía DEA, mientras que para el modelo de FE, la variable dependiente es el resultado promedio de las pruebas Saber Pro. 
tiene mayor repercusión en las pruebas Saber Pro si el máximo grado obtenido es doctorado.

En la educación de los padres, los resultados son variables. Se puede apreciar que a medida que los padres de los estudiantes tienen un mayor nivel de educación, los resultados son mejores. Ahora bien, para el caso del signo negativo en la educación del padre, se debe a que varían los resultados en las pruebas Saber Pro en estas IES, lo cual es coherente con Carella (2009).

Siguiendo con las variables socioeconómicas, se encuentra que el tamaño del hogar es una variable consistente en ambos métodos y disminuye el resultado esperado de las pruebas.

El valor de la matrícula es, quizá, la variable más consistente en la estimación de eficiencia y de frontera estocástica. Para las IES privadas, el valor de la matrícula tendrá una derivación en mejores resultados del examen de calidad educativa. Así mismo, en las IES públicas, el valor de la matrícula disminuye el rendimiento académico. En cuanto al estado civil, se evidencia que tiene una implicación de menor magnitud, pero mejora los resultados promedio de las pruebas.

No obstante, el análisis de FE tiene una connotación mayor, que está dada por el análisis de las desviaciones que produce el error compuesto. Es evidente que tanto para las IES privadas como para las públicas, las mayores desviaciones se deben a la ineficiencia de los factores productivos $(\mathbf{U})$, aunque la ineficiencia en la asignación de sus recursos es mayor en las universidades privadas, al igual que en el modelo DEA.

De otro lado, los errores causados por los factores externos $(\mathrm{V})$ son, en promedio, menores en las IES públicas. Así mismo, cuando se analiza el factor exógeno de la calidad académica, se debe tener en cuenta que la probabilidad de obtener buenos resultados en las pruebas gracias a la suerte es relativamente bajo, dado que se supone que se mide la capacidad de interpretar y plantear soluciones asociadas a un proceso de aprendizaje que dura de cuatro a cinco años en promedio. 
Tabla 5. Fronteras estocásticas para IES privadas.

\begin{tabular}{|l|c|c|}
\hline & Privadas & Publicas \\
\hline Constante & 3.9986 & 4.0100 \\
\hline & $(-0.0657)$ & $(0.0000)$ \\
\hline
\end{tabular}

\section{A. Docentes}

\begin{tabular}{|c|c|c|}
\hline \multirow[t]{2}{*}{ Con maestria } & $0.0006 * *$ & $0.0051 * *$ \\
\hline & $(0.0028)$ & $(0.0008)$ \\
\hline \multirow[t]{2}{*}{ Con doctorado } & $0.0026 * *$ & -0.0169 \\
\hline & $(0.0055)$ & $(0.000)$ \\
\hline \multicolumn{3}{|l|}{ B. Estudiantes } \\
\hline \multirow[t]{2}{*}{ Mujeres } & -0.195 & -0.0064 \\
\hline & $(0.0095)$ & $(0.0000)$ \\
\hline \multirow[t]{2}{*}{ Hombres } & 0.0060 & 0.0083 \\
\hline & $(0.0084)$ & $(0.0000)$ \\
\hline \multicolumn{3}{|c|}{ C. Educacion de los padres } \\
\hline \multirow[t]{2}{*}{ Padres } & $-0,0113$ & $0.0223^{*}$ \\
\hline & $(0.0134)$ & $(0.0000)$ \\
\hline \multirow[t]{2}{*}{ Madre } & $0.0568 * *$ & $0.0835 *$ \\
\hline & $(0.0137)$ & $(0.0000)$ \\
\hline \multicolumn{3}{|l|}{ D. Socio-economico } \\
\hline \multirow[t]{2}{*}{ Tamaño de hogar } & 0.0211 & $-0.1262 * *$ \\
\hline & $(0.0139)$ & $(0.0000)$ \\
\hline \multirow[t]{2}{*}{ Valor de la matricula } & $0.0020^{* * * *}$ & $-0,0003 * *$ \\
\hline & $(0.0014)$ & $(0.0000)$ \\
\hline \multirow{2}{*}{ Estado civil } & $0.0007 * *$ & $0.0144 * *$ \\
\hline & $(0.0082)$ & $(0.0000)$ \\
\hline \multirow[t]{2}{*}{ Personas a cargo } & $-0,0186^{* * * *}$ & $-0,0103^{* * *}$ \\
\hline & $(0.0106)$ & $(0.0000)$ \\
\hline \multirow[t]{2}{*}{ Sigma_V } & $0.0171 *$ & $0.0003^{* *}$ \\
\hline & $(0.0139)$ & $(0.000)$ \\
\hline \multirow[t]{2}{*}{ Sigma_U } & $0.2044^{*}$ & $0.1003^{* *}$ \\
\hline & $(0.0207)$ & $(0.0102)$ \\
\hline \multirow[t]{2}{*}{$\operatorname{Sigma}^{\wedge} 2$} & $(0.0421)^{* * *}$ & $0.0100 *$ \\
\hline & $(0.0081)$ & $(0.0020)$ \\
\hline
\end{tabular}

Fuente: cálculos propios en base a resultados DEA.

Nota: Los resultados tienen como variable dependiente los resultados de la prueba Saber-pro.

Error estándar entre paréntesis, * significativo al $1 \%, * * 5 \%, * * * 10 \%$ 


\section{Conclusiones}

Las IES más eficientes en términos de aprovechamiento de recursos son las universidades y las instituciones universitarias, tanto públicas como privadas.

Se encontró evidencia que permite asegurar que la eficiencia promedio de las IES en Colombia es de $72 \%$ calculado por el DEA y de $87 \%$ mediante la técnica de fronteras estocásticas. La variación del 15\% se debe a factores externos como el clima, la suerte, el estado de ánimo, entre otros, los cuales generan desviaciones en la eficiencia de las IES para garantizar profesionales de excelente calidad.

Después de llevar a cabo el análisis DEA y de frontera, es posible determinar que de 281 IES, solo el $16 \%$ (45) son eficientes.

En cuanto a los determinantes de los resultados de la calidad académica de las distintas IES, es posible afirmar que aquellas que presentan mejor calificación de su planta docente son en promedio más eficientes que aquellas con profesores menos calificados; así mismo, las IES con estudiantes en mayoría mujeres son en promedio más eficientes que aquellas en las cuales predominan los hombres.

Se evidencia que el valor de la matrícula en las IES públicas resulta determinante para la eficiencia de la educación superior en Colombia; no obstante, es necesario ser cuidadosos al analizar este resultado. Un aumento en el valor de la matrícula reduce la calidad académica de los estudiantes, lo cual se puede atribuir a que las IES públicas tienden a exigir mayor tiempo de estudio y esto restringe el ingreso a la vida laboral ${ }^{23}$. A su vez, cuando la matrícula aumenta, se hace necesario que el estudiante acuda al mercado laboral para poder sortear los nuevos costos, lo que conlleva dedicar menos tiempo al estudio.

Otro aspecto importante en la consecución de buenos resultados tiene que ver con el tamaño del hogar, puesto que aquellas IES en las cuales los hogares de los estudiantes son en promedio más grandes también obtienen resultados más bajos.

Resulta relevante la evidencia empírica encontrada por medio de las FE, la cual es muy similar a la encontrada en el modelo Tobit, lo que reafirma que variables como el nivel educativo de los docentes y de los padres, y los aspectos socioeconómicos de los estudiantes determinan la calidad académica de las IES.

23 No se está diciendo que todos los estudiantes de IES públicas solo estudien. Se asegura que el tiempo que demanda la IES pública es en promedio mayor que el de una universidad privada, la cual por lo general tiene programas diurnos y nocturnos. 
La calidad educativa en Colombia depende, en su mayoría, de la ineficiencia de las IES en la asignación de factores productivos; los errores a causa de factores exógenos son en promedio mayores en las universidades privadas.

Implementar políticas enfocadas en la gratuidad del servicio educativo para todos los estudiantes de las IES públicas sería, entonces, un buen paso en la generación de eficiencia relativa mayor.

\section{Recomendaciones}

Optimizar los resultados de calidad de la educación superior colombiana debe ser una prioridad. Debido a los resultados obtenidos, Colombia debe enfocar la política educativa en el mejoramiento de la eficiencia de las IES técnico-profesionales y tecnológicas. De hecho, se debe mejorar la eficiencia individual desde la primera etapa de formación.

Con base en los resultados obtenidos, se hacen las siguientes recomendaciones en pro del mejoramiento de la calidad educativa en Colombia:

Primero, implementar una medición comparativa anual que permita hacer un seguimiento de la variación en los índices de eficiencia por IES. Adicional al examen Saber Pro, es necesario generar mediciones de eficiencia académica que involucren un análisis de corte socioeconómico y financiero de los estudiantes, las cuales pueden fomentar cambios estructurales en los determinantes de la calidad de las IES en Colombia como los encontrados en este estudio.

Segundo, mejorar la calidad educativa desde los niveles básicos. Se sugiere que los colegios públicos del país incluyan dentro de su normativa la obligatoriedad de materias de carácter técnico o tecnológico. Así mismo, debido a que los individuos responden a incentivos, se plantea que todo aquel estudiante que desee seguir en un programa tecnológico y que cumpla con estándares básicos de calidad (medida por un examen tipo Icfes) pueda acceder a programas técnicos especializados con descuento considerable en el costo de la matrícula, esto con el fin de garantizar el acceso a la educación superior y una mayor eficiencia.

Por otra parte, dado que se evidencia que entre los principales determinantes de la calidad educativa, los factores socioeconómicos tienen repercusión a la hora de garantizar mejor eficiencia, se recomienda la creación de una Dirección de Control, resultante de una sinergia entre el Ministerio de Trabajo y el Ministerio de Educación, que permita vigilar, aconsejar y ayudar a los estudiantes (técnicos y tecnólogos) a tener un empleo acorde con su campo educativo, garantizando una remuneración mínima en créditos educativos y en salario; y asimismo, aquellos que empleen a estos estudiantes recibirían exenciones tributarias o beneficios. Trabajos como el de Carella (2009) han encontrado 
evidencia que demuestra cómo aquellos estudiantes que trabajan en lo mismo que estudian son más eficientes.

Tercero, implementar filtros de ingreso a las IES y filtros de grado. Si bien los programas de acceso a la educación por medio de las instituciones del Estado se han visto sopesados por mejores rendimientos, se debe generar una política para mejorar el nivel de eficiencia y eficacia al ingreso a las IES, y que les permita a los estudiantes perfilarse en lo que realmente les interesa y en la demanda interna del país, esto por medio del acompañamiento de psicólogos y expertos en mercado laboral. Adicionalmente, es necesario hacer más restrictivo el proceso de adquisición del diploma, tanto en promedio académico como en niveles de eficiencia individual medidos por técnicas como los datos de panel.

Para próximas investigaciones, se recomienda lo siguiente: 1) Hacer análisis de docentes con doctorado en IES públicas, debido a los resultados no significativos que fueron encontrados; 2) Contrastar las tasas de cobertura y los costos de las matrículas y cómo esta relación afecta los índices de eficiencia técnica; 3) Estudiar la eficiencia de las mujeres en la educación superior y en la retribución que tienen al momento de la inclusión laboral, de modo que se puedan evidenciar los problemas de contratación del país; 4) Evaluar modelos dinámicos que permitan contrastar los resultados aquí encontrados (debido a la naturaleza del trabajo, se invita a utilizar un modelo de ventana del DEA, normalizado con un Tobit o un modelo de datos de panel que permita ver cambios estructurales en la política educativa).

Por último, esta investigación pretende ser un punto de partida en la generación de documentos enfocados en la eficiencia técnica y económica de las IES, así como en el cálculo de mediciones específicas dentro de cada facultad, debido a la generación de estadísticas que permitan identificar los problemas propios de la IES. 


\section{Referencias}

Battese, G. E. y Corra, G. S. (1977). Estimation of a production frontier model: with application to the pastoral zone of Eastern Australia. Australian Fournal of Agricultural Economics, 21(3), 169-179.

Bets, J. D y Morell, D. (1999). The determinants of undergraduate grade point average: the relative importance of family background, high school resources, and peer group effects. The Journal of Human Resources, 34(2), 268-293.

Bessent, A. M y Bessent, E. W. (1980). Determining the comparative efficiency of schools through data envelopment analysis. Educational Administration Quarterly, 16(2), 57-75.

Carrela, L. F. (2009). Educación universitaria: medición del rendimiento académico a través de fronteras de eficiencia (Tesis de maestría, Universidad Nacional de la Plata, La Plata, Argentina. Recuperado de http://www.depeco.econo.unlp.edu.ar/maestria/tesis/069-tesiscarella.pdf

Chalos, P. (1997). An examination of budgetary inefficiency in education using data envelopment analysis. Financial Accountability and Management, 13, 55-69.

Charnes A., Cooper, W. y Rhodes, E. (1978). Measuring the efficiency of decision making units. European Fournal of Operational Research, 2(6), 429-444.

Coleman J. S., Campbell, E., Hobson, C., McPartland J., Mood, A., Weinfeld, F. y York, R. (1966). Equality of educational opportunity. Washington: US Government Printing Office.

Colombia, Congreso de la República. Ley 30, Por la cual se organiza el servicio público de la educación superior (18 diciembre 1992).

Coria, M. M. (2008). Eficiencia técnica de las universidades de gestión estatal en Argentina. Documento de trabajo n. ${ }^{\circ}$ 27. Facultad de Ciencias Sociales y Económicas. Pontificia Universidad Católica Argentina, Buenos Aires. Recuperado de http://www.uca.edu.ar/uca/common/ grupo83/files/27-_Coria.pdf

Departamento Nacional de Planeación (DNP). (2005). Medición y análisis del desempeño integral de los municipios, Informe de resultados vigencia 2005. Bogotá: Autor.

Farell, M. J. (1957). The measurement of productive efficiency. Fournal of the Royal Statistical Society, 120(3), 253-290.

Forero, N. y Ramírez, M. (2008). Determinantes de los ingresos laborales de los graduados universitarios durante el periodo 2001-2004. Documento de trabajo n. ${ }^{\circ} 31$. Facultad de Economía. 
ISSN: 0124-3551 / Año 16, No 24 / enero-junio / pp. 161-194

Universidad del Rosario, Bogotá. Recuperado de http://www.urosario.edu.co/economia/documentos/pdf/dt31.pdf

García, A. P. y González, M. (2013). La evaluación de la eficiencia de las universidades públicas de Colombia utilizando el análisis envolvente de datos (AED). Santander: Universidad Industrial de Santander. Recuperado de http://repositorio.uis.edu.co/jspui/ handle/123456789/7194

González, M. (2010). Análisis de eficiencia y productividad de las universidades chilenas mediante análisis y encapsulamiento de datos. Revista Aporte Santiaguino, 3(2), 245-256. Recuperado de http://www.scielo.org.pe/pdf/as/v3n2/a14v3n2.pdf

Greene, W. H. (2002). Econometric analysis. Nueva York: Prentice Hall.

Hanushek, E. A. (1986). The economics of schooling: production and efficiency in public schools. Fournal of Economic Literature, 24(3), 1141-1177. Recuperado de http:// hanushek.stanford.edu/sites/default/files/publications/Hanushek $\% 201986 \% 20$ JEL $\% 2024 \% 283 \% 29$.pdf

. (1989). The impact of differential expenditure on school performance. Educational Researcher, 45(4), 45-62.

. (1991). When school finance "reform" may not be good policy. Harvard fournal on Legislation, 28(2), 423-456.

. (1996). School resources and student performance. En G. Burtless (ed.). Does money matter? The effect of school resources on student achievement and adult success. Washington: Brookings Institution Press.

. (1998). The evidence on class size. Occasional Paper Number 98-1. W. Allen Wallis Institute of Political Economy, University of Rochester. Recuperado de http:/ /www. wallis.rochester.edu/WallisPapers/wallis_10.pdf

Hoxby, C. (1998). The effect of class size and composition on student achievement: new evidence from natural population variation. NBER Working Paper n. ${ }^{\circ} 6869$. Recuperado de http://www.nber.org/papers/w6869

De Ibarreta, C. M., Rúa, A., Redondo, R., Fabra, M. E., Núñez, A. y Martín M. J. (2009). Influencia del nivel educativo de los padres en el rendimiento académico de los estudiantes de la ADE. Un enfoque de género. Documento de trabajo, Universidad Pontificia Comillas.

Krueger, A. y Whitmore, D. (1999). The effect of attending a small class in the early grades on college - test taking and middle school test results: evidence from Project STAR. Working Paper n. ${ }^{\circ} 427$. Industrial Relations Section, Universidad de Princeton, Nueva Jersey. Recuperado de 
http://dataspace.princeton.edu/jspui/bitstream/88435/dsp010v838058q/1/427. pdf

Lee, C. y Yong-Bae, J. (2010). Data Envelopment Analysis. Stata fournal, 10(2), 267-280.

McCarty, T. A. y Yaisawamg, S. (1993). Technical efficiency in New Jersey school districts. En H. O. Fried, G. A. Lowell y S. S. Schmidt, (eds.). The measurement of productive efficiency: techniques and applications. Nueva York: Oxford University Press.

Miranda J. C. y Araya, L. (2003). Eficiencia económica en las escuelas del MECE/ RURAL desde la perspectiva del análisis envolvente de datos (DEA). Estudios Pedagógicos, 29, 27-39. Recuperado de http://www.scielo.cl/scielo.php?script=sci_ar ttext\&pid=S07 18-07052003000100002

Murias, P., Martínez, R. F., De Miguel, J. C. y Rodríguez, D. (2008). Un estudio con análisis envolvente de datos de la eficiencia de los centros de educación secundaria gallegos. XVI fornadas Asepuma-IV Encuentro Internacional, 16(1), 205.

Muro, J. de D. (1984). Estimación de fronteras de producción: sinopsis y comentarios, Estadística Española, (102), 69-88.

Neylor, R. A. y Smith, J. (2004). The determinants of educational success in higher education. En G. Johnes y J. Johnes (eds.). International handbook of the economics education. Northamptom: Edward Elgar Publishing.

Pérez, H. E., Laguado, A.C. y Martínez, G. (2002). Perfil socioeconómico de los estudiantes de la Universidad Nacional. Estadísticas e indicadores de la Universidad Nacional de Colombia, (6), 190-201. Recuperado de http://www.bdigital.unal.edu.co/3670/9/10_ Perfil_socioecon $\%$ C3\%B3mico_de_los_estudiantes_de_la_Universidad_Nacional_ de_Colombia_2_2A.PDF

Porto, A., Gresia, L. y López, M. (2004). Mecanismos de admisión a la universidad y rendimiento de los estudiantes. La Plata: Universidad Nacional de la Plata. Recuperado de http:// www.depeco.econo.unlp.edu.ar/semi/semi100904.pdf

Quesada, V. M., Blanco, I. y Maza, F.J. (2010). Análisis envolvente de datos aplicado a la cobertura educativa en el departamento de Bolívar-Colombia (2007-2008). Omnia, 16(3), 77-100.

Seiford, L. M. y Thrall, R. M. (1990). Recent developments in DEA. The mathematical programming approach to frontier analysis. Fournal of Econometrics, 46(1), 7-38. 


\section{"Ćl'F́: 24}

ISSN: 0124-3551 / Año 16, No 24 / enero-junio / pp. 161-194

Sengupta, J. K. (1987). Production frontier estimation to measure efficiency: a critical evaluation in light of data envelopment analysis. Managerial and Decision Economics, $8(2), 93-99$.

Smith P.y Mayston D. (1987). Measuring efficiency in the public sector. Omega. International Journal of Management Science, 15(3), 181-189.

Torres-Becerril, O. U., Álvarez, I. y Nava, R. M. (2012). Frontera tecnológica y eficiencia técnica de la educación superior en Colombia. Revista Mexicana de Investigación Educativa, 17(54), 793-816. 